\title{
Oxidative Stress in Early Life: Associations with Sex, Rearing Conditions, and Parental Physiological Traits in Nestling Pied Flycatchers
}

\author{
Jimena López-Arrabé ${ }^{1, *}$ \\ Alejandro Cantarero ${ }^{1}$ \\ Lorenzo Pérez-Rodríguez ${ }^{1,2}$ \\ Antonio Palma ${ }^{1,2}$ \\ Juan Moreno ${ }^{1}$ \\ ${ }^{1}$ Departamento de Ecología Evolutiva, Museo Nacional de \\ Ciencias Naturales, Consejo Superior de Investigaciones \\ Científicas, C/José Gutiérrez Abascal 2, 28006 Madrid, Spain; \\ ${ }^{2}$ Departamento de Ecología Evolutiva, Estación Biológica de \\ Doñana, Consejo Superior de Investigaciones Científicas, \\ Avenida Américo Vespucio s/n, Isla de la Cartuja, 41092 \\ Sevilla, Spain
}

Accepted 12/22/2015; Electronically Published 2/9/2016

\begin{abstract}
Conditions experienced during juvenile development can affect the fitness of an organism. During early life, oxidative stress levels can be particularly high as a result of the increased metabolism and the relatively immature antioxidant system of the individual, and this may have medium- and long-term fitness consequences. Here we explore variation in levels of oxidative stress measured during early life in relation to sex, rearing conditions (hatching date and brood size), and parental condition and levels of oxidative markers in a wild population of the pied flycatcher (Ficedula hypoleuca) followed for 2 yr. A marker of total antioxidant status (TAS) in plasma and total levels of glutathione (GSH) in red blood cells, as well as a marker of oxidative damage in plasma lipids (malondialdehyde [MDA]), were assessed simultaneously. Our results show that nestling total GSH levels were associated with parental oxidative status, correlating negatively with maternal MDA and positively with total GSH levels of both parents, with a high estimated heritability. This suggests that parental physiology and genes could be determinants for endogenous components of the antioxidant system of the offspring. Moreover, we found that total GSH levels were higher in female than in male nestlings and that hatching date was positively associated with antioxidant defenses (higher TAS and total GSH levels). These results suggest that different components of oxidative balance are related to a variety of environmental and intrinsic-including parental-influencing fac-
\end{abstract}

${ }^{*}$ Corresponding author; e-mail: jimena.lopez@mncn.csic.es.

Physiological and Biochemical Zoology 89(2):83-92. 2016. (C) 2016 by The University of Chicago. All rights reserved. 1522-2152/2016/8902-5060\$15.00. DOI: $10.1086 / 685476$ tors. Future experimental studies must disentangle the relative contribution of each of these on nestling oxidative status and how the resulting oxidative stress at early phases shape adult phenotype and fitness.

Keywords: environmental conditions, total glutathione (GSH), heritability, malondialdehyde (MDA), total antioxidant status (TAS), early-life effects.

\section{Introduction}

Oxidative stress-the imbalance between the rate of production of reactive oxygen and nitrogen species and the antioxidant machinery-may affect several fitness-related traits, shaping animal life-history evolution (Halliwell and Gutteridge 2007; Costantini 2008; Dowling and Simmons 2009; Monaghan et al. 2009; Costantini et al. 2010; Metcalfe and Alonso-Alvarez 2010). Juvenile development is a life stage in which oxidative stress levels can be particularly high (Gaál et al. 1996; Nussey et al. 2009), as a result of the high rate of metabolism required for growth, which an immature antioxidant system has difficultly matching (Monaghan et al. 2009; Metcalfe and Alonso-Alvarez 2010 and references therein). Such early oxidative stress during early development may have long-lasting fitness consequences later in life (Blount et al. 2003; Alonso-Alvarez et al. 2006, 2007; Norte et al. 2009; Koivula et al. 2011; Noguera et al. 2011; reviews in Dowling and Simmons 2009; Monaghan et al. 2009; Costantini 2014). Therefore, identifying the main factors determining oxidative status during early development is essential to obtain an integral understanding of life-history trade-offs.

The level of oxidative stress experienced by an organism can be influenced by a combination of genetic and environmental factors. Evidence of a genetic contribution to the generation of reactive oxidative metabolites and resistance to oxidative stress has been provided for a few model species (Costantini and Dell'Omo 2006; Kim et al. 2010; Losdat et al. 2014). The comparison between related individuals (i.e., parents and offspring) allows inferring of the relative contribution of genes to the variability of a specific trait (Falconer and Mackay 1996). This provides an estimate of the narrow sense heritability $\left(h^{2}\right)$, which is defined as the ratio of the additive genetic variance to the phenotypic variance (Lynch and Walsh 1998).

Several studies also support the existence of a contribution of environment to the level of oxidative stress experienced by individuals during early life stages (e.g., Costantini et al. 2006; 
Rubolini et al. 2006; Monaghan 2008; Bourgeon et al. 2011; Lushchack 2011; Stier et al. 2014). For altricial birds, nestrelated conditions determine an immediate environment that influences nestling oxidative stress (e.g., Costantini and Dell'Omo 2006; Costantini et al. 2006; Losdat et al. 2014; López-Arrabé et al. 2015). The number of siblings in the nest, for instance, affects the amount of food received by nestlings, their growth rate, and the energy spent on competition. This could lead to a higher metabolism rate with an overproduction of free radicals and a differential antioxidant intake and allocation, which could ultimately influence nestling oxidative stress (Costantini et al. 2006; Alonso-Alvarez et al. 2007; but see Losdat et al. 2010). In turn, the quantity/quality of the food provided by parents ultimately depends on the productivity of the environment, which is well known to vary seasonally (Martin 1987) and can alter antioxidant status either directly (Blount et al. 2003; Costantini 2010) or by modifying growth trajectories (Alonso-Alvarez et al 2006, 2007). Thus, breeding date is also a critical factor determining offspring fitness, particularly so in migratory species that face opposed environmental constraints between arriving and breeding too early, when weather conditions are still inadequate, and breeding too late, when odds of success decrease and food availability declines (Brown and Brown 2000). However, the effects of these two aspects of rearing conditions on nestling oxidative stress remains understudied.

Here we explore sources of variation in oxidative stress experienced by nestlings relative to the body condition and amount of oxidative stress in parents, nestling sex, and rearing conditions (brood size, hatching date) in a wild population of the migratory pied flycatcher (Ficedula hypoleuca). Some recent studies have explored the sources of variation of oxidative stress in adult pied flycatchers (e.g., Morales et al. 2008, 2011, $2013 b, 2013 c$; López-Arrabé et al. 2014b). However, although there is evidence of associations between nestling oxidative stress and some environmental factors, such as pollutants (Berglund et al. 2007; Rainio et al. 2013; López-Arrabé et al. $2014 a$ ) or the presence of nest-dwelling ectoparasites (LópezArrabé et al. 2015), no comprehensive study exploring patterns in oxidative markers of nestlings has been conducted in this species.

In order to properly evaluate the redox balance of individuals, measures of antioxidant capacity and oxidative damage must be obtained simultaneously (Costantini and Verhulst 2009; Monaghan et al. 2009; Pérez-Rodríguez 2009). Here we use plasma malondialdehyde (MDA) levels - a by-product of lipid peroxidation (Halliwell and Gutteridge 2007) — as a measure of oxidative damage (Mateos et al. 2005; Halliwell and Gutteridge 2007; Sepp et al. 2012). To monitor antioxidant defenses, we use two independent markers: total antioxidant status (TAS) of plasma and total glutathione (GSH) levels in red blood cells (RBCs). TAS measures the capacity of plasma samples to inhibit a redox reaction induced by free radicals (Miller et al. 1993; Cohen et al. 2007) and is primarily the result of the pooled effect of all extracellular antioxidant compounds of the blood (Costantini 2011). Glutathione is a tripeptide thiol functioning in the protection of cells against free radicals, often considered as one of the most important intracellular antioxidants (Meister 1991; Wu et al. 2004). Both TAS and MDA levels can be affected by the concentration of uric acid and triglycerides, respectively, which may confound the interpretation of these oxidative stress biomarkers (Cohen et al. 2007; Costantini 2011; Pérez-Rodríguez et al. 2015). Uric acid is the main form of nitrogen excretion in birds and an indicator of amino acid catabolism, but it is also a powerful antioxidant frequently positively related to TAS values (Cohen et al. 2007; Hõrak et al. 2007; Pérez-Rodríguez et al. 2008a). On the other hand, triglyceride levels can be related to MDA levels, either because of an effect of diet (MDA is also present in food) or because MDA may also be influenced by the amount of circulating lipids susceptible to oxidation (PérezRodríguez et al. 2015). Therefore, we also quantified these two plasma metabolites in order to statistically control for their effects in the analyses.

Our main goal was to examine factors affecting oxidative status in nestlings shortly before fledging, addressing the potential contribution of genetic, parental, and environmental effects. We explored oxidative damage and antioxidants in relation to (1) maternal and paternal oxidative status and body condition, (2) sex of nestlings, and (3) hatching date and brood size. We found that levels of the cellular antioxidant GSH were related to parental oxidative status - showing a significant heritability - but were also influenced by hatching date. We discuss the importance of these links in the context of life history, focusing on the potential impact of oxidative stress during nestling development on individual fitness.

\section{Methods}

\section{General Field Methods}

The study was conducted during spring of 2012 and 2013 in a montane forest of Pyrenean oak, Quercus pyrenaica Willd, at $1200 \mathrm{~m}$ above sea level in Valsaín, central Spain $\left(40^{\circ} 54^{\prime} \mathrm{N}\right.$, $\left.4^{\circ} 01^{\prime} \mathrm{W}\right)$. In the general study area, there are 570 nest boxes (for dimensions, structure, and placement of nest-boxes, see appendix in Lambrechts et al. 2010), around 100 of which are yearly occupied by pied flycatchers.

We followed breeding activities from nest construction to fledging in nest boxes occupied by pied flycatchers. Egg laying in our population of pied flycatchers typically began in late May. Females laid on average six eggs, and chicks usually fledged at the age of $17 \mathrm{~d}$.

We captured adult males and females in their nest boxes while they were provisioning 7-8-d-old nestlings, ringed them if necessary, blood sampled, weighed, and measured them. Body mass was obtained with a digital scale to the nearest $0.1 \mathrm{~g}$, and tarsal length was measured with a digital calliper (precision $0.01 \mathrm{~mm}$ ). We took a blood sample of about $120 \mu \mathrm{L}$ from the brachial vein that was collected in heparinized microcapillaries. We collected blood from adult females during 2012 and $2013(n=34)$ but males only in $2013(n=31)$. Blood samples were stored in Eppendorf tubes in an icebox until re- 
turning to the lab on the same day. In the lab, plasma samples were centrifuged ( $10 \mathrm{~min}$ at $12,000 \mathrm{rpm}$ ) to separate blood cells from plasma, and then both fractions were stored at $-80^{\circ} \mathrm{C}$. If hemolysis occurs during sampling, a possible efflux of intracellular pro-oxidants and antioxidant molecules into plasma could alter levels of oxidative markers, thereby confounding interpretation of results. Thus, hemolysis levels in plasma samples were noted by a visual inspection of red color of plasma samples. We scored samples from 0 (no hemolysis) to 2 (high degree of hemolysis). Only one person scored the degree of hemolysis in order to minimize interobserver variability.

For both years, on day 13 (hatching date $=$ day 1 ), nestlings were ringed, weighed, and measured, and a blood sample was collected following the same protocol as in adults. In 2012, we collected blood from all chicks in the nest $(n=199)$, but in 2013, we took blood samples from two randomly selected nestlings of each nest $(n=74)$.

\section{Lipid Peroxidation Assay}

Plasma concentrations of MDA were analyzed as described by López-Arrabé et al. (2014b). Briefly, a standard curve was prepared for calibration, using a 1,1,3,3-tetraethoxypropane stock solution serially diluted in $40 \%$ ethanol. Butylated hydroxytoluene, phosphoric acid, and thiobarbituric acid (TBA) solutions were added to each plasma sample and standard. Then, samples were incubated on a dry bath to allow formation of MDA-TBA adducts. After that, pure n-butanol was added to each sample and standard. Tubes were vortexed and centrifuged, and the upper phase was collected and transferred into a high-performance liquid chromatography (HPLC) vial for analysis. Samples were injected into an Agilent 1200 HPLC system (Agilent Technologies, Santa Clara, CA). Data were collected using a fluorescence detector (G1321A, Agilent Technologies). Repeatability (following Lessells and Boag 1987), calculated on a set of samples assayed in duplicate, was high ( $r=0.722, N=66, P<0.001)$. Interassay coefficient of variability $(\mathrm{CV})$ was $8.08 \%$.

\section{Total Antioxidant Status (TAS)}

TAS was analyzed as described by López-Arrabé et al. (2014b). As standard for the assays, we used Trolox (a water-soluble $\alpha$-tocopherol derivative), and TAS levels are expressed in Trolox-equivalent units. The assays were run on a Synergy HT Multi-Mode Microplate Reader (BioTek Instruments, Winooski, VT). In order to accurately control the reaction time, only one column of the plate was used at a time. To standard or samples were sequentially added metmyoglobin (a mixed of equal volumes of myoglobin [M0630-250MG, Sigma-Aldrich, St. Louis] and potassium ferricyanate), ABTS (the chromogen, 2,2'-azino-bis-(3-ethylbenzothiazoline-6-sulphonic acid)), and $\mathrm{H}_{2} \mathrm{O}_{2}$, starting the reaction. Kinetic measurements were immediately started, recording absorbance at $660 \mathrm{~nm}$ every $5 \mathrm{~s}$. The temperature was maintained at $37^{\circ} \mathrm{C}$ during assays. All samples were assayed in duplicate, and results showed a high repeatability $(r=0.981, N=234, P<0.001)$ and an interassay CV of $3.63 \%$.

\section{Intracellular Total GSH Level}

Total GSH levels in RBCs were determined as described by López-Arrabé et al. (2014a) and Romero-Haro and AlonsoAlvarez (2015). Briefly, RBC samples were diluted and homogenized in a stock buffer and mixed with trichloroacetic acid. The mixture was vortexed and centrifuged, and the supernatant was separated. The next steps were performed on a Synergy HT Multi-Mode Microplate Reader (BioTek Instruments). To samples (supernatant) we added a mixture of nicotinamide adenine dinucleotide phosphate and 5,5'-dithiobis(2-nitrobenzoic acid). Afterward, a GSH reductase solution was added after $15 \mathrm{~s}$, and the absorbance at $405 \mathrm{~nm}$ was monitored after 15 and $45 \mathrm{~s}$. The change in absorbance was used to determine the intracellular tGSH concentration by comparing the output with the results from a standard curve generated by serial dilution of GSH. The assays were performed at $37^{\circ} \mathrm{C}$, and only one column of the plate was used at a time in order to control reaction times accurately. A set of samples assayed in duplicate showed a high repeatability $(r=$ $0.983, N=106, P<0.001)$ and an interassay CV of $3.89 \%$.

\section{Measurement of Uric Acid and Triglyceride Levels}

Following previous studies in birds (e.g., Pérez-Rodríguez et al. 2008a, 2015; Romero-Haro and Alonso-Alvarez 2015), plasma levels of uric acid and triglycerides were measured using commercial kits (11522 and 11529 from Biosystems, Barcelona) based on the uricase/peroxidase method (Fossati et al. 1980) and the glycerol phosphate oxidase/peroxidase method (Bucolo et al. 1973), respectively. Analyses were run in 96-well plates using the same microplate reader mentioned before. For uric acid, $150 \mu \mathrm{L}$ of the chromogen were added to $5 \mu \mathrm{L}$ of each plasma sample or the standard (a $6 \mathrm{mg} / \mathrm{dL}$ uric acid solution). For triglycerides, $250 \mu \mathrm{L}$ of the chromogen were added to $5 \mu \mathrm{L}$ of each plasma sample or the standard (a $200 \mathrm{mg} / \mathrm{dL}$ glycerol solution). In both cases, plates were incubated for $5 \mathrm{~min}$ at $37^{\circ} \mathrm{C}$, subsequently measuring absorbance at $520 \mathrm{~nm}$ (for uric acid) or $500 \mathrm{~nm}$ (for triglycerides). A subset of samples assayed in duplicate showed a high repeatability for both variables (uric acid: $r=0.99, P<0.001$; triglycerides: $r=0.94, P<0.001 ; N=45$ in both cases). Interassay CVs were $2.79 \%$ and $3.76 \%$ for uric acid and triglycerides, respectively.

\section{Sex Determination}

The sex of nestlings was determined by the amplification of the CHD sequence present in both $\mathrm{W}$ and $\mathrm{Z}$ avian chromosomes, by polymerase chain reactions (PCRs) using the primers P2 (5'-TCT GCA TCG CTA AAT CCT TT-3') and P8 (5'-CTC CCA AGG ATG AGR AAY TG-3'; Griffiths et al. 1998). We 
performed assays on a GeneAmp PCR System 9700 (Applied Biosystems, Foster City, CA). Reaction conditions were as follows: an initial denaturing step at $94^{\circ} \mathrm{C}$ for $4 \mathrm{~min}$ and $30 \mathrm{~s}$ was followed by 45 cycles of $94^{\circ} \mathrm{C}$ for $30 \mathrm{~s}, 49^{\circ} \mathrm{C}$ for $45 \mathrm{~s}$, and $72^{\circ} \mathrm{C}$ for $45 \mathrm{~s}$. A final run of $72^{\circ} \mathrm{C}$ for 5 min completed the program. PCR products were separated by electrophoresis in cyan/yellow loading buffer (Invitrogen, Life Technologies, Carlsbad, CA) at $100 \mathrm{~V}$ for $20 \mathrm{~min}$. The amplification products corresponded to CDHW and CDHZ genes. Males are identified by having only the $\mathrm{CDHZ}$ band, while females present both $\mathrm{CDHZ}$ and $\mathrm{CDHW}$ bands.
Statistical Analyses

In order to statistically control for potentially confounding effects, we explored the effect of the degree of hemolysis on the oxidative stress parameters of both nestlings and adults. For nestlings and adult males, hemolysis affected only MDA levels (both $P<0.001)$. For adult females, hemolysis affected MDA $(P<0.001)$ and total GSH $(P=0.032)$ levels. In these cases, we controlled the variables-when entered either as dependent variables or as covariates-by the degree of hemolysis. Also, we ran models restricted to nonhemolyzed samples in

Table 1: Results of mixed models exploring associations between physiological variables of nestling and female pied flycatchers (Ficedula hypoleuca)

\begin{tabular}{|c|c|c|c|c|}
\hline & Estimate $\pm \mathrm{SE}$ & df & $F$ & $P$ \\
\hline \multicolumn{5}{|l|}{ Nestling MDA $(\mu \mathrm{mol} / \mathrm{mL})$ : } \\
\hline \multicolumn{5}{|l|}{ Full model: } \\
\hline Hatching date & $.006 \pm .014$ & $1,37.0$ & .19 & 665 \\
\hline Brood size & $-.044 \pm .049$ & $1,44.8$ & .83 & .367 \\
\hline Female body condition & $.064 \pm .056$ & $1,39.2$ & 1.33 & .255 \\
\hline Female hemolysis-corrected MDA & $.011 \pm .010$ & $1,33.2$ & 1.30 & .261 \\
\hline Female uric acid-corrected TAS & $-.225 \pm .132$ & $1,57.4$ & 2.90 & .094 \\
\hline Female hemolysis-corrected total GSH & $.071 \pm .072$ & $1,41.5$ & .96 & .332 \\
\hline Hemolysis & $.396 \pm .053$ & $1,114.0$ & 55.73 & $<.001^{\star}$ \\
\hline Year & $.055 \pm .163$ & $1,39.5$ & .11 & .737 \\
\hline \multicolumn{5}{|l|}{ Final model: } \\
\hline Hemolysis & $.370 \pm .044$ & $1,251.0$ & 71.05 & $<.001^{\star}$ \\
\hline \multicolumn{5}{|l|}{ Nestling TAS (mmol/L): } \\
\hline \multicolumn{5}{|l|}{ Full model: } \\
\hline Hatching date & $.017 \pm .020$ & $1,15.9$ & .75 & .400 \\
\hline Brood size & $.001 \pm .068$ & $1,18.1$ & .00 & .993 \\
\hline Female body condition & $-.096 \pm .087$ & $1,17.5$ & 1.21 & .286 \\
\hline Female hemolysis-corrected MDA & $.027 \pm .015$ & $1,16.5$ & 3.42 & .082 \\
\hline Female uric acid-corrected TAS & $-.117 \pm .170$ & $1,37.4$ & .47 & .495 \\
\hline Female hemolysis-corrected total GSH & $-.016 \pm .103$ & $1,18.5$ & .03 & .874 \\
\hline Uric acid & $.113 \pm .013$ & $1,73.6$ & 71.92 & $<.001^{\star}$ \\
\hline Year & $-.011 \pm .230$ & $1,16.7$ & .00 & .961 \\
\hline \multicolumn{5}{|l|}{ Final model: } \\
\hline Hatching date & $.017 \pm .006$ & $1,59.3$ & 7.00 & $.010^{\star}$ \\
\hline Uric acid & $.116 \pm .008$ & $1,169.0$ & 226.37 & $<.001^{\star}$ \\
\hline \multicolumn{5}{|l|}{ Nestling total GSH $(\mu \mathrm{mol} / \mathrm{g})$ : } \\
\hline \multicolumn{5}{|l|}{ Full model: } \\
\hline Hatching date & $.002 \pm .034$ & $1,28.4$ & .00 & .953 \\
\hline Brood size & $-.012 \pm .115$ & $1,33.0$ & .01 & .915 \\
\hline Female body condition & $-.179 \pm .132$ & $1,28.7$ & 1.83 & .187 \\
\hline Female hemolysis-corrected MDA & $-.024 \pm .023$ & $1,24.8$ & 1.02 & .322 \\
\hline Female uric acid-corrected TAS & $-.258 \pm .302$ & $1,50.3$ & .73 & .397 \\
\hline Female hemolysis-corrected total GSH & $.539 \pm .170$ & $1,30.1$ & 10.01 & $.003^{\star}$ \\
\hline Year & $-.029 \pm .389$ & $1,29.6$ & .01 & .941 \\
\hline \multicolumn{5}{|l|}{ Final model: } \\
\hline Female hemolysis-corrected MDA & $-.035 \pm .016$ & $1,55.5$ & 4.80 & $.033^{\star}$ \\
\hline Female hemolysis-corrected total GSH & $.279 \pm .102$ & $1,83.5$ & 7.42 & $.008^{\star}$ \\
\hline
\end{tabular}

Note. GSH, glutathione; MDA, malondialdehyde; TAS, total antioxidant status.

*Significant difference $(\alpha=0.05)$. 


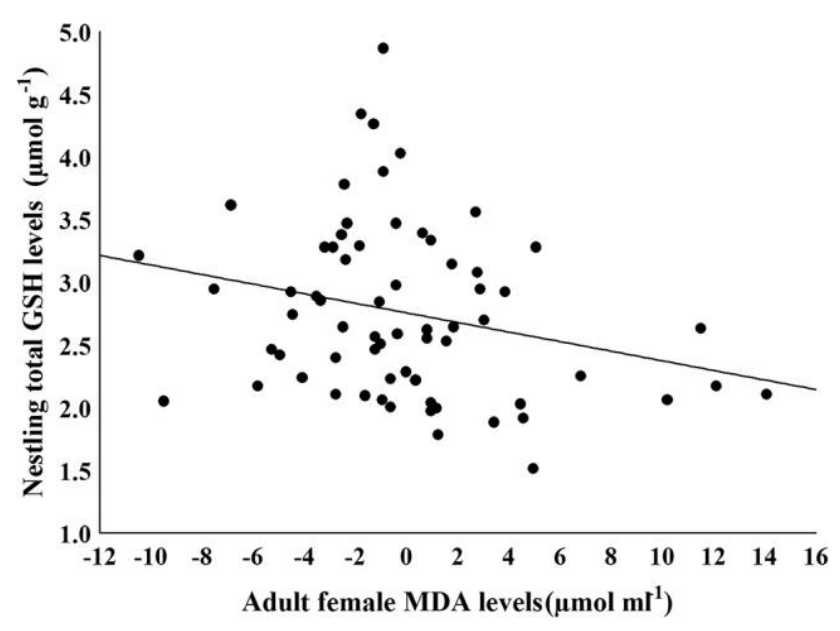

Figure 1. Relation between nestling total glutathione (GSH) levels and adult female malondialdehyde (MDA) levels, corrected by hemolysis degree $\left(F_{1,64}=4.36, P=0.041\right)$ in the pied flycatcher (Ficedula hypoleuca).

order to assess the robustness of our results. Uric acid affects plasma TAS measures (Cohen et al. 2007; Pérez-Rodríguez et al. 2008b), so this marker of antioxidant capacity was always controlled for uric acid levels when entered in the models. Also, as recently proposed (Pérez-Rodríguez et al. 2015), statistical models for MDA were run including and excluding triglyceride level as a covariate.

To analyze the relationships between nestling oxidative stress parameters and parental oxidative status and body condition, we ran separate models including nestling MDA, TAS, or total GSH as dependent variables and either female or male parent oxidative stress measures and body condition (residuals of the regression of body mass on tarsus length) as covariates. In all cases, we controlled for hatching date and brood size by including them as covariates. In models analyzing associations with the oxidative status and body condition of the female parent, we also included year of sampling as fixed factor. For data from 2013, we estimated heritability $\left(h^{2}\right)$ through midparent-offspring correlations (Falconer and Mackay 1996). The slope of the regression of mean offspring measurements on the mean of their parent's measurements (the midparent value) constitutes a crude measure of heritability when full pedigrees are not available. We have assumed that paternity has been accurately estimated through paternal care at the nest, an assumption that could introduce some error if extra-pair paternity was high in 2013 (for estimates of extra-pair paternity in the population, see Moreno et al. 2013a, 2015).

Also, we analyzed the relationships between nestling oxidative stress parameters and breeding variables (hatching date and brood size) by running models that included nestling MDA, TAS, or total GSH as dependent variables; hatching date, brood size, and nestling body condition as covariates; and sex of nestlings and year of sampling as fixed factors.

Finally, to test the potential relationship between nestling body condition and breeding variables, we ran a model including body mass as a dependent variable; hatching date, brood size, and tarsus length as covariates; and year as a fixed factor.

In all cases, we used mixed models using Proc Mixed in SAS (ver. 9.3) and restricted maximum likelihood to analyze the variation in nestling oxidative variables. Degrees of freedom (df) were estimated using the Satterthwaite approximation, thus avoiding possible pseudoreplication. Only nestling MDA levels were log transformed to obtain a normal distribution. The rest of dependent variables were normally distributed. Nest was included as a random factor to avoid pseudoreplication, and individual nestling was used as a sample unit. Moreover, in those cases (only three) in which adult females were captured in the $2 \mathrm{yr}$ of the study, data from one of the years randomly were eliminated to avoid pseudoreplication. To obtain minimal adequate models, we elaborated full saturated models including in each case the factors mentioned above, and we sequentially removed the less significant terms following a standard backward stepwise procedure until only significant variables were retained.

\section{Results}

We found a negative association between total GSH levels in nestlings and adult female MDA (table 1; fig. 1). Moreover, total GSH levels in nestlings and adult females were significantly and positively correlated (table 1). A similar positive association was found between nestling and male total GSH levels $\left(F_{1,27.6}=7.35, P=0.011\right.$; estimate $\left.=0.440 \pm 0.162\right)$. Heritability of total GSH levels varied from 0.60 to 0.71 (fig. 2). Although they were separated by less than $2 \mathrm{SD}$ from the mean of the sample, two potential outliers might influence results (fig. 2). However, when we repeated the analyses excluding them, the results remained the same $\left(F_{1,24}=15.74\right.$, $P \leq 0.001 ; y=0.3628+0.7071 x)$.

Nestlings that hatched later in the breeding season showed higher levels of total GSH (table 2) and higher TAS values

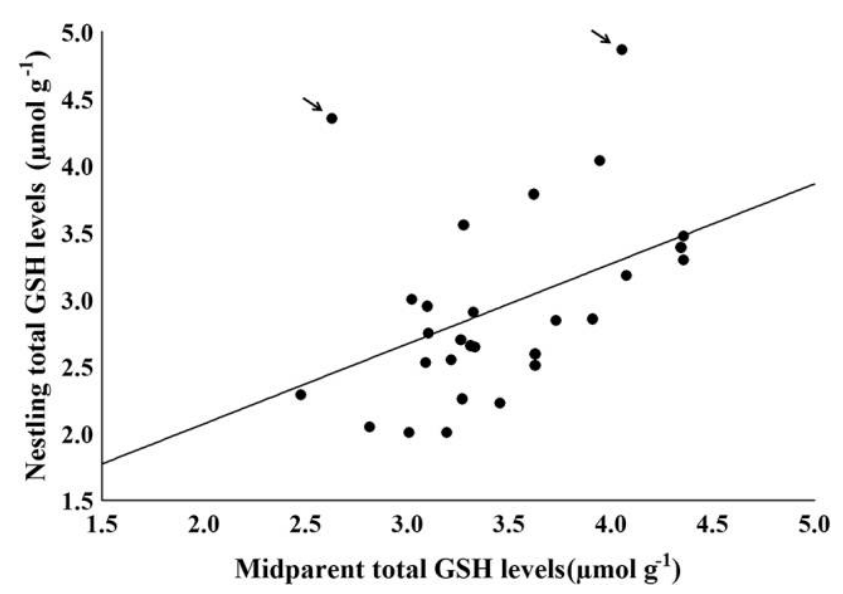

Figure 2. Relation between midparent and mean nestling pied flycatcher (Ficedula hypoleuca) total glutathione (GSH) levels in 2013 $\left(F_{1,26}=5.78, P=0.024 ; y=0.8758+0.5973 x\right)$. Arrows indicate possible outliers (see "Results"). 
Table 2: Results of mixed models exploring associations among physiological variables and breeding variables (brood size and hatching date) of nestling pied flycatchers (Ficedula hypoleuca)

\begin{tabular}{|c|c|c|c|c|}
\hline & Estimate \pm SE & df & $F$ & $P$ \\
\hline \multicolumn{5}{|l|}{$\operatorname{MDA}(\mu \mathrm{mol} / \mathrm{mL}):$} \\
\hline \multicolumn{5}{|l|}{ Full model: } \\
\hline Hatching date & $.014 \pm .009$ & $1,111.0$ & 2.33 & .129 \\
\hline Brood size & $.042 \pm .034$ & $1,105.0$ & 1.52 & .221 \\
\hline Sex & $.014 \pm .045$ & $1,230.0$ & .10 & .749 \\
\hline Body condition & $.018 \pm .027$ & $1,208.0$ & .44 & .507 \\
\hline Hemolysis & $.372 \pm .044$ & $1,244.0$ & 69.84 & $<.001^{\star}$ \\
\hline Year & $.105 \pm .104$ & $1,129.0$ & 1.03 & .311 \\
\hline \multicolumn{5}{|l|}{ Final model: } \\
\hline Hemolysis & $.371 \pm .044$ & $1,250.0$ & 70.53 & $<.001^{\star}$ \\
\hline \multicolumn{5}{|l|}{ TAS (mmol/L): } \\
\hline \multicolumn{5}{|l|}{ Full model: } \\
\hline Hatching date & $.006 \pm .010$ & $1,75.6$ & .35 & .558 \\
\hline Brood size & $-.020 \pm .036$ & $1,76.9$ & .32 & .571 \\
\hline Sex & $.014 \pm .043$ & $1,136.0$ & .11 & .735 \\
\hline Body condition & $-.014 \pm .027$ & $1,179.0$ & .25 & .616 \\
\hline Uric acid & $.116 \pm .008$ & $1,156.0$ & 214.15 & $<.001^{\star}$ \\
\hline Year & $-.149 \pm .107$ & $1,104.0$ & 1.95 & .166 \\
\hline \multicolumn{5}{|l|}{ Final model: } \\
\hline Uric acid & $.116 \pm .008$ & $1,160.0$ & 231.41 & $<.001^{*}$ \\
\hline Year & $-.218 \pm .071$ & $1,85.9$ & 9.34 & $.003^{*}$ \\
\hline \multicolumn{5}{|l|}{ Total GSH $(\mu \mathrm{mol} / \mathrm{g})$ : } \\
\hline \multicolumn{5}{|l|}{ Full model: } \\
\hline Hatching date & $.028 \pm .020$ & $1,102.0$ & 1.98 & .163 \\
\hline Brood size & $-.056 \pm .071$ & $1,92.7$ & .61 & .435 \\
\hline Sex & $-.186 \pm .085$ & $1,238.0$ & 4.81 & $.029^{*}$ \\
\hline Body condition & $.025 \pm .052$ & $1,234.0$ & .24 & .628 \\
\hline Year & $-.215 \pm .213$ & $1,126.0$ & 1.02 & .315 \\
\hline \multicolumn{5}{|l|}{ Final model: } \\
\hline Hatching date & $.045 \pm .012$ & $1,81.4$ & 12.83 & $<.001^{\star}$ \\
\hline Sex & $-.185 \pm .084$ & $1,240.0$ & 4.80 & $.029^{\star}$ \\
\hline
\end{tabular}

Note. GSH, glutathione; MDA, malondialdehyde; TAS, total antioxidant status.

${ }^{\star}$ Significant difference $(\alpha=0.05)$.

(table 1). Moreover, total GSH levels were significantly higher in female than in male nestlings (table 2; fig. 3). Finally, there were no associations of nestling body condition with brood size or hatching date (both $P>0.06$ ).

None of the above mentioned results for MDA levels (tables 1,2 ) changed when plasma triglyceride levels were entered as a covariate in the respective models. In the same way, results were qualitatively similar when we restricted our analyses to nonhemolyzed samples.

\section{Discussion}

We have searched for possible associations of nestling and parental oxidative variables and body condition in a pied flycatcher population. Moreover, we explored variation in levels of oxidative stress in nestlings in relation to sex and rearing conditions (hatching date and brood size). Our results showed that nestling total GSH levels were negatively associated with maternal MDA and positively associated with total GSH levels of both parents, also showing a high estimated heritability. Moreover, total GSH levels were higher in female than in male nestlings, and hatching date was positively associated with antioxidant defenses.

The negative association between nestling total GSH and MDA levels of the female parent could reflect costs and/or constraints derived from parental care. Females experiencing lower levels of oxidative damage could be able to invest more resources in their offspring, for example, by increasing provisioning rates and/or supplying food items of higher nutritive value to nestlings. This could have a positive effect on nestling oxidative status, as reflected by higher constitutive levels of a key antioxidant, such as GSH. Alternatively, poorly fed nestlings would beg more intensely (Redondo and Castro 1992; Cantarero et al. 2013), which would increase their level of oxidative stress (increased oxidative damage and/or depleted antioxidant defenses; Moreno-Rueda et al. 2012). This could 


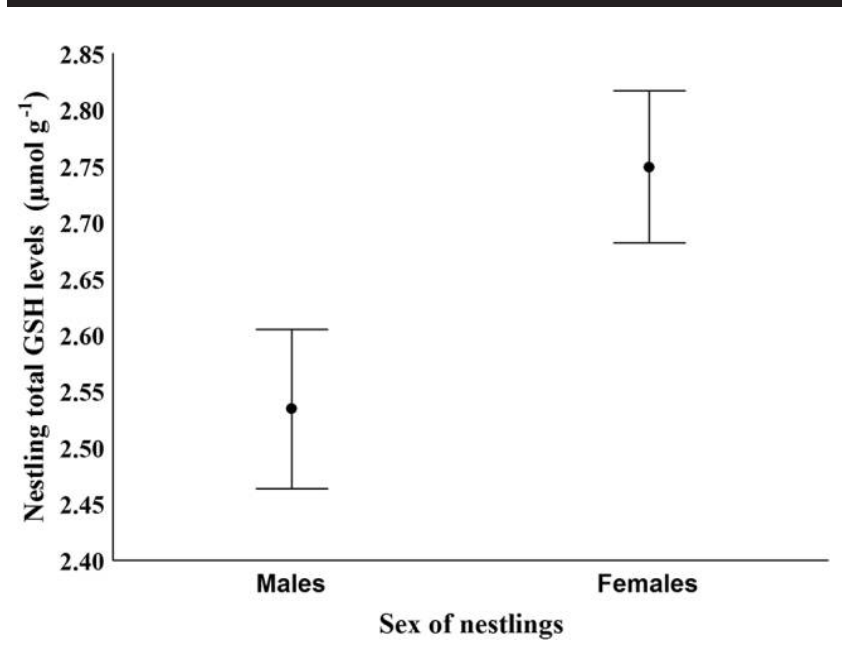

Figure 3. Differences $\left(F_{1,270}=4.83, P=0.029\right)$ in total glutathione (GSH) levels (mean \pm SE) between female and male nestling pied flycatchers (Ficedula hypoleuca).

elicit a behavioral response from parents, thereby increasing parental effort (Cantarero et al. 2013), which could increase metabolic rate and oxidative stress (Nilsson 2002; AlonsoAlvarez et al. 2004; Wiersma et al. 2004).

The strong positive correlation detected between parentoffspring total GSH levels could result from the relationship between parental oxidative status and parental effort and its subsequent effect on nestling antioxidant depletion. However, the high value of $h^{2}$ estimated for this trait suggests that the levels of this crucial antioxidant compound could be under strong genetic control. Similarly, a high heritability of GSH enzymes has been found for humans (60\%; Chakraborty and Chaudhuri 2001). The antioxidant role of GSH in RBCs depends on the rates of synthesis and degradation of GSH but also on the activity of enzymes involved in GSH action (Halliwell and Gutteridge 2007; Lu 2009). However, GSH is also involved in other important cellular processes not directly linked to antioxidant protection, such as regulation of DNA synthesis, melanogenesis, providing a reservoir for cysteine, or regulating cell growth and death ( $\mathrm{Lu}$ 2009). A strong genetic control of total GSH levels would help to buffer sudden changes in antioxidant requirements, assuring an optimal GSH supply to satisfy those alternative functions of this molecule.

Unlike total GSH, we found no heritability for TAS and MDA levels. TAS is a measure of the levels of nonenzymatic antioxidants - such as vitamins A, C, and E-and carotenoids (Cohen et al. 2007), many of which are obtained from the diet. Thus, TAS could reflect parental ability to find high-quality food as well as other environmental factors affecting nutrient availability and allocation. Similarly, levels of MDA could be substantially influenced by environmental conditions, since oxidative damage reflects the balance between production of pro-oxidants and levels of antioxidant molecules, including those contributing to TAS (Costantini 2014). Consistent with this, a recent study has also shown a high environmental contribution to MDA levels in nestlings of another passerine (Losdat et al. 2014). The lack of parent-offspring correlations in TAS and MDA levels could be also explained by the stronger effect of some rearing conditions (e.g., diet, nest ectoparasites; Rubolini et al. 2006; de Coster et al. 2012; López-Arrabé et al. 2015; Wegmann et al. 2015) in the nestlings as compared with adults.

It should be mentioned, however, that our conclusions on trait heritabilities must be taken with caution because they are based on midparent-offspring regressions, which could be influenced by shared environmental conditions that can affect similarities shown between relatives (Kruuk and Hadfield 2007). The application of restricted maximum likelihood animal models to data from natural populations offers a powerful means of tackling these potentially confounding effects (Kruuk 2004). Unfortunately, in our case, we had data from only two generations, which makes individual pedigrees unreliable.

Associations found between nestling antioxidant defenses (TAS and total GSH) and hatching date could be explained by seasonal changes in the availability of specific nutrients and/ or the metabolic constraints faced by the developing individual (Biard et al. 2005; Sternalski et al. 2010). Although early breeding is usually associated with higher parental and nestling quality (Verhulst and Nilsson 2008 and references therein), it could also result in increased physiological stress (Lobato et al. 2010) and metabolic costs as a result of cold weather conditions under which parental foraging efficiency is low (Stevenson and Bryant 2000). Moreover, cooler microclimate conditions in the nest-as found in our study area early in the reproductive season-may impose higher metabolic costs for nestlings (Dawson et al. 2005), which may have a direct negative impact on the oxidative status of nestlings that hatch earlier through depleting their antioxidant reserves (Bourgeon et al. 2011).

Interestingly, we found significant differences in total GSH levels between nestling sexes, with these being higher in females than in males. Similar results have been shown for other species (Isaksson 2013). This sexual difference is likely to be mediated via testosterone levels, because male nestlings often show higher circulating levels of this hormone than females (Naguib et al. 2004; Müller et al 2007; Kozlowski and Ricklefs 2011). Higher concentrations of testosterone could induce higher levels of oxidative stress (e.g., Alonso-Alvarez et al. 2007, 2009; Mougeot et al 2009), leading to a faster decrease of total GSH in males as compared with females.

In conclusion, we have evaluated several natural correlates of oxidative stress levels in pied flycatcher nestlings, and we have found that sex and breeding time explain part of the variation in oxidative status, since antioxidant defenses were higher in female chicks and late-hatched broods. Moreover, we suggest that parental oxidative status and genes could be determinants for endogenous components of the nestlings antioxidant system, like total GSH levels. It has been demonstrated that early conditions can influence trade-offs and may program the individual phenotype throughout the life- 
time (Lindström 1999; Metcalfe and Monaghan 2001; RomeroHaro and Alonso-Alvarez 2015). Understanding the relative contribution of genotype and environmental conditions to the oxidative stress experienced by the developing nestling - as well as the life-history trade-offs associated with the interaction between both factors - is essential to fully understand the importance of oxidative stress in shaping individual phenotype.

\section{Acknowledgments}

This study was financed by project CGL2013-48193-C3-3-P to J.M. from the Spanish Ministerio de Economía y Competitividad (MINECO). A.C. was supported by a Formación de Personal Universitario grant from the Spanish Ministerio de Educación, Cultura y Deporte, and J.L.-A. was supported by an a Formación de Personal Investigador grant from MINECO. L.P.-R. was supported by a postdoctoral contract from MINECO through the Severo Ochoa Programme for Centres of Excellence in Research, Development, and Innovation (SEV-2012-0262). Permission for handling birds was provided by the Consejería de Medio Ambiente de Castilla y León, and J. Donés and M. Redondo of Centro Montes de Valsaín allowed us to work in the study area. We thank S. Merino, E. Pérez-Badás, J. Rivero-de Aguilar, and A. DíezFernández for collaboration in the field. We are also grateful to J. D. Blount for initial advice on the analysis of malondialdehyde levels. This study is a contribution to the research developed at El Ventorrillo field station. The study was approved by the Ethical Committee of the Consejo Superior de Investigaciones Científicas and by the regional administration competent in matters related to animal protection, according to Royal Decree 53/2013 (Dirección General de Producción Agropecuaria y Desarrollo Rural, Junta de Castilla y León, Spain).

\section{Literature Cited}

Alonso-Alvarez C., S. Bertrand, G. Devevey, J. Prost, B. Faivre, O. Chastel, and G. Sorci. 2006. An experimental manipulation of life-history trajectories and resistance to oxidative stress. Evolution 60:1913-1924.

Alonso-Alvarez C., S. Bertrand, G. Devevey, J. Prost, B. Faivre, and G. Sorci. 2004. Increased susceptibility to oxidative stress as a proximate cost of reproduction. Ecol Lett 7:363368.

Alonso-Alvarez C., S. Bertrand, B. Faivre, and G. Sorci. 2007. Increased susceptibility to oxidative damage as a cost of accelerated somatic growth in zebra finches. Funct Ecol 21: 873-879.

Alonso-Alvarez C., L. Pérez-Rodríguez, J.T. Garcia, and J. Viñuela. 2009. Testosterone-mediated trade-offs in the old age: a new approach to the immunocompetence handicap and carotenoid-based sexual signalling. Proc R Soc B 276: 2093-2101
Berglund A., J. Sturve, L. Förlin, and N.E.I. Nyholm. 2007. Oxidative stress in pied flycatcher (Ficedula hypoleuca) nestlings from metal contaminated environments in northern Sweden. Environ Res 105:330-339.

Biard C., P.F. Surai, and A.P. Møller. 2005. Effects of carotenoid availability during laying on reproduction in the blue tit. Oecologia 144:32-44.

Blount J.D., N.B. Metcalfe, K.E. Arnold, P.F. Surai, G.L. Devevey, and P. Monaghan. 2003. Neonatal nutrition, adult antioxidant defences and sexual attractiveness in the zebra finch. Proc R Soc B 270:1691-1696.

Bourgeon S., S. Guindre-Parker, and T.D. Williams. 2011. Effects of sibling competition on growth, oxidative stress, and humoral immunity: a two-year brood-size manipulation. Physiol Biochem Zool 84:429-437.

Brown C.R. and M.B. Brown. 2000. Weather-mediated natural selection on arrival time in cliff swallows (Petrochelidon pyrrhonota). Behav Ecol Sociobiol 47:339-345.

Bucolo G. and H. David. 1973. Quantitative determination of serum triglycerides by use of enzymes. Clin Chem 19:476482.

Cantarero A., J. López-Arrabé, A.J. Redondo, and J. Moreno. 2013. Behavioural responses to ectoparasites in pied flycatchers Ficedula hypoleuca: an experimental study. I Avian Biol 44:591-599.

Chakraborty S. and A.B.D. Chaudhuri. 2001. Heritability of some important parameters of the antioxidant defense system like glucose-6-phosphate dehydrogenase, catalase, glutathione peroxidase and lipid peroxidation in red blood cells by twin study. Int J Hum Genet 1:229-232.

Cohen A., K. Klasing, and R. Ricklefs. 2007. Measuring circulating antioxidants in wild birds. Comp Biochem Physiol B $147: 110-121$.

Costantini D. 2008. Oxidative stress in ecology and evolution: lessons from avian studies. Ecol Lett 11:1238-1251.

- 2010. Effects of diet quality on serum oxidative status and body mass in male and female pigeons during reproduction. Comp Biochem Phvsiol A 156:294-299.

- 2011. On the measurement of circulating antioxidant capacity and the nightmare of uric acid levels. Methods Ecol Evol 2:321-325.

. 2014. Oxidative stress and hormesis in evolutionary ecology and physiology. Springer, Berlin.

Costantini D., S. Casagrande, S. De Filippis, G. Brambilla, A. Fanfani, J. Tagliavini, and G. Dell'Omo. 2006. Correlates of oxidative stress in wild kestrel nestlings (Falco tinnunculus). I Comp Physiol B 176:329-337.

Costantini D. and G. Dell'Omo. 2006. Environmental and genetic components of oxidative stress in wild kestrel nestlings (Falco tinnunculus). L Comp Phvsiol B 176:575-579.

Costantini D., M. Rowe, M.W. Butler, and K.J. McGraw. 2010. From molecules to living systems: historical and contemporary issues in oxidative stress and antioxidant ecology. Funct Ecol 24:950-959.

Costantini D. and S. Verhulst. 2009. Does high antioxidant capacity indicate low oxidative stress? Funct Ecol 23:506-509. 
Dawson R.D., C.C. Lawrie, and E.L. O’Brien. 2005. The importance of microclimate variation in determining size, growth and survival of avian offspring: experimental evidence from a cavity nesting passerine. Oecologia 144:499-507.

de Coster G., L. de Neve, S. Verhulst, and L. Lens. 2012. Maternal effects reduce oxidative stress in female nestlings under high parasite load. L Avian Biol 43:177-185.

Dowling D.K. and L.W. Simmons. 2009. Reactive oxygen species as universal constraints in life-history evolution. Proc R Soc B 276:1737-1745.

Falconer D.S. and T.F.C. Mackay. 1996. Introduction to quantitative genetics. Longmans Green, Harlow.

Fossati P., L. Prencipe, and G. Berti 1980. Use of 3,5-dichloro-2hydroxybenzenesulfonicacid/4-aminophenazone chromogenic system in direct enzymic assay of uric acid in serum and urine. Clin Chem 26:227-231.

Gaál T., B.K. Speake, M. Mezes, R.C. Noble, P.F. Surai, and P. Vajdovich. 1996. Antioxidant parameters and ageing in some animal species. Comp Hematol Int 6:208-213.

Griffiths R., M.C. Double, K. Orr, and R.J. Dawson. 1998. A DNA test to sex most birds. Mol Ecol 7:1071-1075.

Halliwell B. and J. Gutteridge. 2007. Free radicals in biology and medicine. Oxford University Press, Oxford.

Hõrak P., L. Saks, M. Zilmer, U. Karu, and K. Zilmer. 2007. Do dietary antioxidants alleviate the cost of immune activation? an experiment with greenfinches. Am Nat 170:625-635.

Isaksson C. 2013. Opposing effects on glutathione and reactive oxygen metabolites of sex, habitat, and spring date, but no effect of increased breeding density in great tits (Parus major). Ecol Evol 3:2730-2738.

Kim S.Y., J.C. Noguera, J. Morales, and A. Velando. 2010. Heritability of resistance to oxidative stress in early life. I Evol Biol 23:769-775.

Koivula M.J., M. Kanerva, J.P. Salminen, M. Nikinmaa, and T. Eeva. 2011. Metal pollution indirectly increases oxidative stress in great tit (Parus major) nestlings. Environ Res 111: 362-370.

Kozlowski C.P. and R.E. Ricklefs. 2011. The effects of brood size on growth and steroid hormone concentrations in nestling eastern bluebirds (Sialia sialis). Gen Comp Endocrinol 173:447-453.

Kruuk L.E. 2004. Estimating genetic parameters in natural populations using the "animal model." Philos Trans R Soc B 359:873-890.

Kruuk L.E.B. and J.D. Hadfield. 2007. How to separate genetic and environmental causes of similarity between relatives. I Evol Biol 20:1890-1903.

Lambrechts M.M., F. Adriaensen, D.R. Ardia, A.V. Artemyev, F. Atiénzar, J. Bánbura, E. Barba, et al. 2010. The design of artificial nestboxes for the study of secondary hole-nesting birds: a review of methodological inconsistencies and potential biases. Acta Ornithol 45:1-26.

Lessells C.M. and P.T. Boag. 1987. Unrepeatable repeatabilities: a common mistake. Auk 104:116-121.

Lindström J. 1999. Early development and fitness in birds and mammals. Trends Ecol Evol 14:343-348.
Lobato E., J. Moreno, S. Merino, J. Morales, G. Tomás, J. Martínez, R.A. Vásquez, A. Kuchar, E. Möstl, and J.L. Osorno. 2010. Arrival date and territorial behavior are associated with corticosterone metabolite levels in a migratory bird. I Ornithol 151:587-597.

López-Arrabé J., A. Cantarero, L. Pérez-Rodríguez, A. Palma, C. Alonso-Alvarez, S. González-Braojos, and J. Moreno. 2015. Nest-dwelling ectoparasites reduce antioxidant defences in females and nestlings of a passerine: a field experiment. Oecologia 179:29-41.

López-Arrabé J., A. Cantarero, L. Pérez-Rodríguez, A. Palma, and J. Moreno. 2014a. Experimental pyrethroid treatment underestimates the effects of ectoparasites in cavity-nesting birds due to toxicity. Ibis 156:606-614.

- 2014b. Plumage ornaments and reproductive investment in relation to oxidative status in the Iberian pied flycatcher (Ficedula hypoleuca iberiae). Can I Zool 92:1019-1027.

Losdat S., F. Helfenstein, J.D. Blount, and H. Richner. 2014. Resistance to oxidative stress shows low heritability and high common environmental variance in a wild bird. LEvol Biol 27:1990-2000.

Losdat S., F. Helfenstein, B. Gaude, and H. Richner. 2010. Effect of sibling competition and male carotenoid supply on offspring condition and oxidative stress. Behav Ecol 21: $1271-1277$.

Lu S.C. 2009. Regulation of glutathione synthesis. Mol Aspects Med 30:42-59.

Lushchak V.L. 2011. Environmentally induced oxidative stress in aquatic animals. Aquat Toxicol 101:13-30.

Lynch M. and B. Walsh. 1998. Genetics and analysis of quantitative traits. Sinauer, Sunderland, MA.

Martin T.E. 1987. Food as a limit on breeding birds: a lifehistory perspective. Annu Rev Ecol Syst 18:453-487.

Mateos R., E. Lecumberri, S. Ramos, L. Goya, and L. Bravo. 2005. Determination of malondialdehyde (MDA) by highperformance liquid chromatography in serum and liver as a biomarker for oxidative stress: application to a rat model for hypercholesterolemia and evaluation of the effect of diets rich in phenolic antioxidants from fruits. $\underline{\text { Chromatogr B }}$ 827:76-82.

Meister A. 1991. Glutathione deficiency produced by inhibition of its synthesis, and its reversal; applications in research and therapy. Pharmacol Ther 51:155-194.

Metcalfe N.B. and C. Alonso-Alvarez. 2010. Oxidative stress as a life-history constraint: the role of reactive oxygen species in shaping phenotypes from conception to death. Funct Ecol 24:984-996.

Metcalfe N.B. and P. Monaghan. 2001. Compensation for a bad start: grow now, pay later? Trends Ecol Evol 16:254-260.

Miller N.J, C. Rice-Evans, M.J. Davies, V. Gopinathan, and A. Milner. 1993. A novel method for measuring antioxidant capacity and its application to monitoring the antioxidant status in premature neonates. Clin Sci 84:407-412.

Monaghan P. 2008. Early growth conditions, phenotypic development and environmental change. Philos Trans R Soc B 363:1635-1645. 
Monaghan P., N.B. Metcalfe, and R. Torres. 2009. Oxidative stress as a mediator of life history trade-offs: mechanisms, measurements and interpretation. Ecol Lett 12:75-92.

Moreno J., J.G. Martínez, S. González-Braojos, A. Cantarero, R. Ruiz-de-Castañeda, M. Precioso, and J. López-Arrabé. 2015. Extra-pair paternity declines with female age and wing length in the pied flycatcher. Ethology 121:501-512.

Moreno J., J.G. Martínez, S. González-Braojos, R. Ruiz-deCastañeda, A. Cantarero, and A. Sánchez-Tójar. 2013a. Extrapair matings, context-dependence and offspring quality: a brood manipulation experiment in pied flycatchers. Behaviour 150: 359-380.

Moreno J., A. Velando, S. González-Braojos, R. Ruiz-deCastañeda, and A. Cantarero. 2013b. Females paired with more attractive males show reduced oxidative damage: possible direct benefits of mate choice in pied flycatchers. Ethology 119: 727-737.

Morales J., A. Velando, and J. Moreno. 2008. Pigment allocation to eggs decreases plasma antioxidants in a songbird. Behav Ecol Sociobiol 63:227-233.

Moreno J., A. Velando, R. Ruiz-De-Castañeda, C. Cantarero, S. González-Braojos, and A. Redondo. 2011. Plasma antioxidant capacity and oxidative damage in relation to male plumage ornamental traits in a montane Iberian pied flycatcher Ficedula hypoleuca population. Acta Ornithol 46:65-70.

Moreno J., A. Velando, R. Ruiz-de-Castañeda, S. GonzálezBraojos, and A. Cantarero. 2013c. Oxidative damage in relation to a female plumage badge: evidence for signalling costs. Acta Ethol 16:65-75.

Moreno-Rueda G., T. Redondo, C.E. Trenzado, A. Sanz, and J.M. Zúñiga. 2012. Oxidative stress mediates physiological costs of begging in magpie (Pica pica) nestlings. PLoS One 7:e40367.

Müller W., K. Deptuch, I. López-Rull, and D. Gil. 2007. Elevated yolk androgen levels benefit offspring development in a between-clutch context. Behav Ecol 18:929-936.

Naguib M., K. Riebel, A. Marzal, and D. Gil. 2004. Nestling immunocompetence and testosterone covary with brood size in a songbird. Proc R Soc B 271:833-838.

Nilsson J.Å. 2002. Metabolic consequences of hard work. Proc R Soc B 269:1735-1739.

Noguera J.C., S.Y. Kim, and A. Velando. 2011. Pre-fledgling oxidative damage predicts recruitment in a long-lived bird. Biol Lett 8:61-63.

Norte A.C., B.C. Sheldon, J.P. Sousa, and J.A. Ramos. 2009. Environmental and genetic variation in body condition and blood profile of great tit Parus major nestlings. L Avian Biol 40:157-165.

Nussey D.H., J.M. Pemberton, J.G. Pilkington, and J.D. Blount. 2009. Life history correlates of oxidative damage in a free-living mammal population. Funct Ecol 23:809-817.

Pérez-Rodríguez L. 2009. Carotenoids in evolutionary ecology: re-evaluating the antioxidant role. Bioessavs 10:11161126.
Pérez-Rodríguez L., C. Alonso-Alvarez, M. Martínez-Haro, and J. Viñuela. 2008a. Variation in plasma biochemical parameters in captive adult red-legged partridges (Alectoris rufa) during daylight hours. Eur I Wild Res 54:21-26.

Pérez-Rodríguez L., F. Mougeot, C. Alonso-Alvarez, J. Blas, J. Viñuela, and G.R. Bortolotti. 2008b. Cell-mediated immune activation rapidly decreases plasma carotenoids but does not affect oxidative stress in red-legged partridges (Alectoris rufa). LExp Biol 211:2155-2161.

Pérez-Rodríguez L., A.A. Romero-Haro, A. Sternalski, J. Muriel, F. Mougeot, D. Gil, and C. Alonso-Alvarez. 2015. Measuring oxidative stress: the confounding effect of lipid concentration in measures of lipid peroxidation. Physiol Biochem Zool 88:345-351.

Rainio M.J., M. Kanerva, J.P. Salminen, M. Nikinmaa, and T. Eeva. 2013. Oxidative status in nestlings of three small passerine species exposed to metal pollution. Sci Total Environ 454:466-473.

Redondo T. and F. Castro. 1992. Signalling of nutritional need by magpie nestlings. Ethology 92:193-204.

Romero-Haro A.A. and C. Alonso-Alvarez. 2015. The level of an intracellular antioxidant during development determines the adult phenotype in a bird species: a potential organizer role for glutathione. Am Nat 185:390-405.

Rubolini D., M. Romano, A. Bonisoli Alquati, and N. Saino. 2006. Early maternal, genetic and environmental components of antioxidant protection, morphology and immunity of yellow-legged gull (Larus michahellis) chicks. LEvol Biol 19:1571-1584.

Sepp T., U. Karu, J.D. Blount, E. Sild, M. Männiste, and P. Hõrak. 2012. Coccidian infection causes oxidative damage in greenfinches. PLoS One 7:e36495.

Sternalski A., F. Mougeot, C. Eraud, B. Gangloff, A. Villers, and V. Bretagnolle. 2010. Carotenoids in nestling Montagu's harriers: variations according to age, sex, body condition and evidence for diet-related limitations. I Comp Physiol B 180:33-43.

Stevenson I.R. and D.M Bryant. 2000. Avian phenology: climate change and constraints on breeding. Nature 406:366-367.

Stier A., S. Massemin, and F. Criscuolo. 2014. Chronic mitochondrial uncoupling treatment prevents acute cold-induced oxidative stress in birds. I Comp Physiol B 184:1021-1029.

Verhulst S. and J.Å. Nilsson. 2008. The timing of birds' breeding seasons: a review of experiments that manipulated timing of breeding. Philos Trans R Soc B 363:399-410.

Wegmann M., B. Boegeli, and H. Richner. 2015. Physiological responses to increased brood size and ectoparasite infestation: adult great tits favour self-maintenance. Physiol Behav 141:127-134.

Wiersma P., C. Selman, J.R. Speakman, and S. Verhulst. 2004. Birds sacrifice oxidative protection for reproduction. Proc $\mathrm{R}$ Soc B 271:S360-S363.

Wu G., Y.Z. Fang, S. Yang, J.R. Lupton, and N.D. Turner. 2004. Glutathione metabolism and its implications for health. L Nutr 134:489-492. 